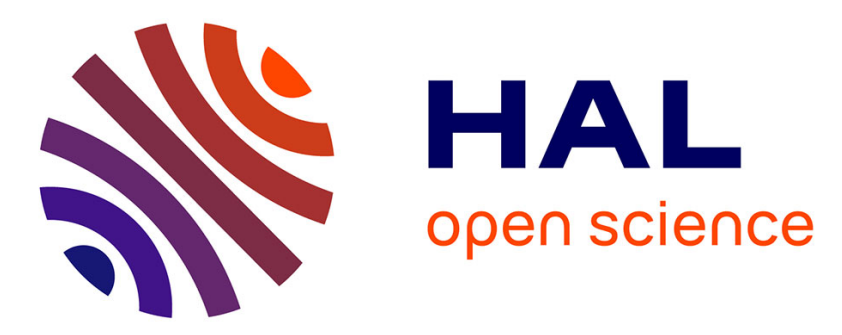

\title{
Capital social et enjeux de pouvoir : une perspective socio-politique de l'appropriation d'une technologie de réseaux sociaux au sein d'une collectivité territoriale
}

Myriam Karoui, Aurélie Dudézert

\section{- To cite this version:}

Myriam Karoui, Aurélie Dudézert. Capital social et enjeux de pouvoir : une perspective socio-politique de l'appropriation d'une technologie de réseaux sociaux au sein d'une collectivité territoriale. Systèmes d'Information et Management, 2012, 17 (1), pp.49-80. 10.3917/sim.121.0049 . hal-00744688

\section{HAL Id: hal-00744688}

https://hal-centralesupelec.archives-ouvertes.fr/hal-00744688

Submitted on 23 Oct 2012

HAL is a multi-disciplinary open access archive for the deposit and dissemination of scientific research documents, whether they are published or not. The documents may come from teaching and research institutions in France or abroad, or from public or private research centers.
L'archive ouverte pluridisciplinaire HAL, est destinée au dépôt et à la diffusion de documents scientifiques de niveau recherche, publiés ou non, émanant des établissements d'enseignement et de recherche français ou étrangers, des laboratoires publics ou privés. 


\section{Capital social et enjeux de pouvoir : une perspective so- cio-politique de l'appropriation d'une technologie de ré- seaux sociaux au sein d'une collectivité territoriale}

\section{Myriam Karoui}

Doctorante Ecole Centrale Paris

Laboratoire Génie Industriel, Equipe EPOCC

Grande voie des Vignes

92295 Châtenay-Malabry cedex

myriam.karoui@ecp.fr

Tél +33(0)141131166

\section{Aurélie Dudezert}

Maître de conférences HDR

Laboratoire Génie Industriel, Equipe EPOCC

Ecole Centrale Paris

Grande Voie des Vignes,

92295 Châtenay-Malabry cedex

aurelie.dudezert@ecp.fr

Tel : +33 (0) 141131605

\section{Biographies des auteurs :}

Myriam KAROUI est doctorante à l'Ecole Centrale Paris dans le domaine du Management des Systèmes d'Information. Au sein de l'équipe EPOCC (Equipe de recherche sur les Politiques de Croissance fondées sur la Connaissance), ses recherches portent sur les médias sociaux, les outils des réseaux sociaux et leur appropriation ainsi que les nouvelles pratiques de travail nées avec l'émergence des outils 2.0.

Aurélie DUDEZERT est Maître de Conférences en Sciences de Gestion Habilitée à Diriger les Recherches à l'Ecole Centrale Paris. Spécialiste du Management des Connaissances elle est aujourd'hui en charge du développement de l'équipe en Sciences Economiques et Sciences de Gestion de 1'Ecole Centrale Paris (Equipe de recherche sur les Politiques de Croissance fondées sur la Connaissance-EPOCC) qui se donne pour objectif d'étudier les nouveaux modèles de croissance de l'économie et les nouveaux modes d'organisation des entreprises à l'heure de l'Economie de la Connaissance. 


\section{口 Résumé}

Parmi les outils 2.0 émergents les technologies dites de réseaux sociaux (Social Network Systems- SNS) sont considérées comme des supports permettant le développement d'un capital social facilitant la collaboration et l'innovation dans les organisations. Dans un environnement économique et managérial global où le capital social est de plus en plus considéré comme un pré-requis à l'action collective en organisation, l'introduction de ces technologies de réseaux sociaux peut être également une opportunité pour les acteurs de l'organisation de redistribuer le pouvoir et l'influence entre eux. L'objectif de ce travail est de comprendre comment les enjeux de pouvoir liés au développement du capital social impactent l'appropriation d'une technologie SNS. A travers une Recherche Action menée au sein d'une Mairie et en s'appuyant sur la théorie de la pratique de Bourdieu et la théorie de l'acteur stratégique de Crozier et Friedberg, nous identifions que l'appropriation de la technologie de réseaux sociaux est principalement une appropriation socio-politique en deux phases : une phase d'appropriation de la nouvelle dimension donnée au capital social et une phase d'appropriation de la technologie pour servir les intérêts des acteurs concernant le développement du capital social. Ce travail met également en lumière le rôle que peuvent jouer les systèmes d'information et notamment les outils de réseaux sociaux dans le maintien et le développement des positions sociales des acteurs au sein de l'organisation.

Mots clefs :

Technologie 2.0, réseau social, SNS, capital social, stratégie d'acteur.

\section{a Abstract}

Emergent tools 2.0 like social networks technologies or Social Networks Systems (SNS) allow the development of a social capital and facilitate collaboration and innovation through organizations. In an economic and managerial environment where social capital is considered as a prerequisite for the collective action in organization, the introduction of these technologies of Social Networks Systems can be also an opportunity for actors to redistribute the power and the influence between them in the organization. This research study aims to understand how the stakes in power related to the development of the social capital can impact the adoption of SNS tool by actors. Through an Action- Research within a City and based on Bourdieu's theory on the social positions and on Crozier and Friedberg's strategic actor's theory, we identify that the appropriation of social networks technology is primarily a socio-political appropriation in two phases: in the first phase actors discover social capital as a new symbolic capital and in a second phase they use the technology to influence the development of the organizational social capital in order it can serve their own interests. This work highlights also the role played by information systems and particularly social network systems in the development and maintaining of actors' social positions within the organization.

Key-words:

Technologies 2.0, social network, SNS, social capital, actors' strategies. 


\section{Introduction}

Depuis 2005, les technologies de réseaux sociaux ou Social Network Systems (SNS) se sont déployées partout dans le monde et ont été adoptées par les internautes avec une vitesse sans égale pour des technologies de l'information. En moins de 8 ans, $12 \%$ de la population mondiale a créé un compte sur le réseau social Facebook, et en Avril 2010 le journal Les Echos soulignait que 100\% des étudiants américains avaient un compte sur ce réseau social. Ces technologies ont aussi accompagnées certaines mutations sociales et politiques de pays émergents et leur rôle a été largement souligné dans la révolution arabe de 2011 par exemple.

Du fait de leur popularité incontestable dans la sphère privée (Roblyer et al., 2010), les outils de réseaux sociaux suscitent de plus en plus l'intérêt du monde scientifique (Pempek et al., 2009 ; Nov, 2009 ; Boyd \& Ellison, 2007 ; Richter et al., 2011) comme des entreprises (Lecko, 2011 ; Manhes, 2011). L'engouement pour ces technologies interroge particulièrement les organisations. Pour la première fois, des technologies sont accessibles aux individus massivement avant qu'elles ne soient prises en main par l'entreprise. Alors que les entreprises ont joué un rôle actif dans la généralisation du téléphone, du fax ou même d'internet comme pratique sociale, dans le cas des technologies web 2.0 et plus particulièrement des technologies de réseaux sociaux, le phénomène a été inversé.

Basés sur la théorie des graphes, ces technologies de réseaux sociaux mettent en exergue les liens formels et informels reliant les collaborateurs et leur permettent d'établir de nouveaux liens pour étendre leur réseau relationnel au sein de l'organisation. Ces technologies sont particulièrement utiles pour faciliter les collaborations inter-services et les relations informelles (Cross, Parker and Borgatti, 2002) et sont de plus en plus vues comme des moteurs de l'innovation pour les organisations (Deltour et al., 2011). Toutefois le capital social étant le réseau des relations interpersonnelles et informelles dans l'organisation (Burt, 1992; 2000; Steinfield, DiMicco, Ellison, and Lampe, 2009), ces technologies rendent aussi visible ce capital social ainsi que la manière dont le travail est réellement réalisé (Cross \& Parker, 2004). La coordination et la réalisation du travail au sein des organisations se font en partie à travers un circuit de relations informelles et implicites en contournant les procédures officielles et les chaines hiérarchiques (Cross et al., 2000). Ce capital social a toujours joué un rôle important pour mener l'action collective de l'organisation. Toutefois dans les organisations actuelles flexibilisées et post-bureaucratiques, il joue un rôle encore plus central (Lazega, 2006) et rendre visible ce qui jusque là ne l'était pas (les réseaux sociaux et la place de ce capital social dans l'organisation) peut avoir des impacts socio-politiques importants au sein de l'organisation (Star \& Bowker, 1995 ; Star \& Strass, 1999). En mettant en évidence les réseaux informels de chaque acteur de l'organisation, les technologies de réseaux sociaux mettent ainsi en évidence le capital social de chaque acteur et peuvent conduire les acteurs de l'organisation à re-négocier leur position sociale. Bien plus, ces outils reposent sur une déclaration individuelle et autonome des relations entre acteurs et de leur position dans un réseau social. En conséquence, les acteurs d'une organisation peuvent être tentés de profiter de l'introduction d'un SNS pour orienter le développement du capital social de l'organisation dans un sens qui sert au mieux leurs propres intérêts (développement de leur propre capital social par exemple) et conforte leur pouvoir au sein de l'organisation. Aussi l'introduction des outils de réseaux sociaux dans les organisations induit des changements majeurs dans la distribution du pouvoir et du contrôle au sein de l'organisation (Buckhardt \& Brass, 1990). 
En adoptant la perspective socio-politique de l'appropriation des technologies (de Vaujany, 2006), cet article étudie dans quelle mesure les enjeux de pouvoir liés au développement du capital social contribuent à l'appropriation d'une technologie SNS. En d'autres termes, ce travail de recherche a pour but de comprendre comment les acteurs s'emparent d'une technologie de réseau social dans un environnement organisationnel et quelle place tiennent les enjeux socio-politiques dans l'appropriation de cet outil. Pour ce faire, nous nous appuyons sur une Recherche Action menée au sein d'une collectivité territoriale française et sur un cadre théorique mobilisant la théorie de l'acteur stratégique de Crozier et Friedberg (1977) et les travaux de Bourdieu $(1994$; 2000) relatifs à la lutte pour le maintien des positions sociales. Cet article est organisé de la sorte : la première partie présente les fondements théoriques de cette recherche. La deuxième partie présente le cadre théorique d'analyse mobilisé par ces travaux. La démarche de Recherche Action au sein de la Mairie est exposée dans la troisième partie. Dans une quatrième et cinquième partie, le cas et son analyse sont présentés. Enfin, la dernière partie discute les limites et les perspectives de ce travail au regard des travaux du champ.

\section{Fondements théoriques : réseau social, capital social et technologies de réseaux sociaux}

Les technologies dites de réseaux sociaux (Social Network Systems- SNS) sont considérées comme des supports pouvant permettre l'animation des réseaux sociaux d'une organisation. Un réseau social correspond à un ensemble de nœuds (individus, organisations) reliés par un ensemble de relations sociales (Fuhrer et al., 2009). Ces relations prennent forme spontanément et sont donc informelles. Ceci étant, ces relations restent importantes à identifier car elles forment un processus social permettant le transfert et le partage des connaissances (Burt 1992, Szulanski 1996) et la localisation de l'expertise (Anklam, 2003). Ces réseaux relationnels permettent d'accélérer le travail collectif dans l'organisation en dépassant les logiques hiérarchiques officielles. Ainsi ces réseaux sont fréquemment mobilisés par les acteurs pour obtenir une information utile ou solliciter l'aide d'un collègue qui a une connaissance nécessaire à la résolution d'un problème sans passer par la hiérarchie. La généralisation de l'email dans les organisations ainsi que l'aplanissement des structures et la disparition de certains niveaux hiérarchiques ont encore accentué ces dernières années la place de ces réseaux dans l'action collective. Ces réseaux sociaux sont à l'origine du développement d'un capital social utile et nécessaire à l'organisation.

Le capital social est l'avantage qu'un individu peut obtenir en étant connecté à d'autres. Comme le soulignent Fuhrer et Cucchi et Picard (2009, p.4), le capital social est considéré dans la littérature comme une ressource bénéficiant à un niveau collectif et individuel et qui «s'appuyant sur des normes, des valeurs partagées et la confiance, facilite la coordination ou la coopération entre les individus ». Le capital social est un phénomène cumulatif par le biais des interactions sociales mises en œuvre dans le réseau social (Putman, 2000 ; Bourdieu, 1980 ; 1985). Il dépend de la taille du réseau relationnel et du capital social des individus qui le compose. Ce capital est construit à travers les relations qu'un individu entretient avec un groupe d'autres individus (Putnam, 1995, 2001). L'appartenance à ce groupe et donc à un réseau social permet aux individus de se constituer un capital social qui leur donne accès à un ensemble d'avantages significatifs (Cross et al., 2002 ; Burton et al., 2010) tels que le pouvoir (Brass, 1984), le leadership (Sparrowe \& Lidden, 1997), la mobilité (Boxman et al., 1991), l'employabilité (Fernandez et al., 2000) et la performance de l'équipe (Burton et al., 2010). 
Au niveau individuel, ce capital social est utile et productif dans la mesure où il permet aux individus qui le mobilisent l'accomplissement de certaines actions. Il peut conduire selon certains auteurs au développement d'un mieux vivre ensemble (Putman, 2000 ; Coleman, 1988), la réalisation de certains objectifs collectifs (Coleman, 1988) mais peut aussi être utilisé comme ressource mobilisée par un individu pour satisfaire ses propres intérêts et accroître son pouvoir (Bourdieu, $1980 ; 1985$ ).

Au niveau organisationnel, comme le soulignent Deltour, Plé et Roussel (2011) ce capital social est de plus en plus considéré comme un pré-requis à l'action collective en organisation. De fait Nahapiet et Ghoshal (1998) mettent en évidence que si le capital social dans sa dimension structurelle renvoie aux connections, liens et hiérarchies du réseau constitué et repose sur des relations fondées sur la confiance et le respect (dimension relationnelle), il produit des représentations partagées et un langage commun (dimension cognitive) fondement d'une action collective dans les organisations.

Deltour, Plé et Roussel (2011) proposent une analyse des potentialités de développement du capital social de l'organisation via les technologies web 2 .0. Ils montrent ainsi que les outils web 2.0 et particulièrement les outils de réseaux sociaux (SNS) contribuent au développement du capital social. Ces technologies ont pour fonctionnalités d'identifier, de représenter, d'analyser et de visualiser les nœuds (par exemple des acteurs, des connaissances, des organisations...) et les relations entre les nœuds. Un outil de réseaux sociaux est un outil qui fait apparaitre de façon visuelle les réseaux sociaux, par exemple en termes de relations entre les personnes. Ces technologies permettent donc à une organisation de mettre en lumière les relations informelles entre agents, de provoquer des opportunités d'échange, de création des connaissances, de visualiser et de comprendre les relations susceptibles d'améliorer la collaboration et d'accroître l'interaction sociale (Cross et al., 2002 ; Pereira \& Soares 2007, Fisher \& Dourish, 2004). Aussi, introduire un outil de socialisation tel qu'un outil de réseaux sociaux peut contribuer au développement du capital social d'une organisation (Borgatti \& Foster, 2003 ; Burt, 2005 ; Burton et al., 2010).

Des travaux récents étudient les usages des technologies de réseaux sociaux par les acteurs de l'entreprise et les types de relations sociales qu'ils priviligient lors de l'utilisation de ces technologies. Ainsi Ferron, Massa et Odella (2011) étudie la communauté de chercheurs de la Fondation Bruno Kessler (FBK) où un réseau social de 400 utilisateurs a été déployé appelé «Taolin Platform ». Ils étudient en particulier le phénomène d'homophilie et analysent le type de connections que les utilisateurs produisent en utilisant l'outil de réseau social. Ils mettent en avant que dans le contexte de la Fondation Bruno Kessler les acteurs utilisent principalement l'outil de réseau social pour re-créer les liens existant dans le monde réel. A l'inverse Steinfield, DiMicco, Ellison et Lample (2009) étudient le cas d'un réseau social déployé pour 30000 utilisateurs chez IBM et appellé «Beehive » et mettent en avant que dans ce contexte les acteurs n'utilisent pas l'outil de réseau social pour maintenir des relations interpersonnelles avec leurs collègues les plus proches mais profitent de ce système pour se connecter à d'autres collègues qu'ils n'ont pas eu l'occasion de connaitre. Ils cherchent alors à développer des liens transversaux, qui dépassent les limites des départements et des directions et qui surtout leur permettent d'etre connectés à d'autres individus ayant les mêmes centres d'intérêt. Néanmoins dans tous ces cas, en mettant en lumière les relations informelles au sein de l'organisation, le SNS change la représentation de la structure organisationnelle en mettant en lumière les pratiques collaboratives et les échanges interpersonnels. Boland, Lyytinen et Yoo (2007) montrent comment un changement dans la représentation numérique de données (digital representations) qui sont cruciales pour le fonctionnement d'une organisation peut engendrer de multiples changements en particulier dans les pratiques de travail. La plupart des organisations sont 
structurées hiérarchiquement avec des procédures formelles. La représentation de ce qu'est l'organisation se fait via l'organigramme. En rendant visibles les relations informelles et interpersonnelles au sein de l'organisation, le SNS change la représentation de la structure organisationnelle: il objective la pratique de travail et peut conduire à interroger la pertinence de la répartition des tâches et du pouvoir dans l'organisation (Star et Strauss, 1999). Cette nouvelle représentation peut par exemple, inciter les acteurs à s'affranchir des logiques hiérarchiques et à privilégier le contact direct entre acteurs. Au-delà, la mise en lumière de nouvelles informations sur l'organisation du travail peut amener à considérer de nouveaux élements de distinction et de comparaison entre acteurs. Par exemple, les acteurs qui sont amenés dans l'exécution de leurs tâches à travailler en collaboration avec d'autres services pourront afficher un réseau social étendu dans l'organisation alors que d'autres dont la tâche est cantonnée à un service ne pourront pas forcément le faire. La visibilité du réseau social de chacun ne bénéficiera pas de la même manière aux acteurs de l'organisation et le système pourra être jugé par les acteurs comme inéquitable (Joshi, 1991). Certains acteurs seront alors réticents à l'utilisation du système pour protéger leurs propres intérêts et leur position sociale dans l'entreprise quand d'autres au contraire seront promoteurs de ce système qui leur donne l'opportunité de définir une nouvelle position sociale. Enfin, ces technologies étant techniquement simples à utiliser et leur fonctionnement reposant sur des données individuelles et personnelles qu'acceptent de déclarer les acteurs, les individus sont libres d'afficher ce qu'ils souhaitent au sein du SNS. Les acteurs exercent leur rationalité et un comportement stratégique lors de l'implémentation d'un SI dans une organisation (Elie-dit-Cosaque, 2010). Aussi comme dans les réseaux sociaux présents sur le web (Cardon, 2008), les acteurs peuvent développer des stratégies au sein de leur organisation pour rendre visible et gérer leur capital social grâce à ces technologies. Ils peuvent choisir de ne pas présenter tout leur réseau social dans le système. Au contraire, ils peuvent choisir de s'auto-promouvoir et de présenter tout type de liens.

Les enjeux de pouvoir et le comportement stratégique des acteurs de l'organisation vis-àvis de la technologie jouent donc un rôle important dans l'appropriation des technologies SNS par les acteurs d'une organisation. Aussi pour étudier l'appropriation d'un tel outil, il paraît pertinent d'adopter la perspective socio-politique de l'appropriation (De Vaujany, 2006) et d'étudier dans quelle mesure les enjeux de pouvoir liés au développement du capital social de l'organisation peuvent impacter l'appropriation d'une technologie SNS. C'est pourquoi cet article s'appuie sur un cadre théorique mobilisant la théorie de l'acteur stratégique de Crozier et Friedberg (1977) et la théorie de la pratique de Bourdieu (1994, 2000).

\section{Le cadre théorique d'analyse}

Pour Crozier et Friedberg (1977), l'acteur est un stratège au sein de l'organisation. Selon eux les acteurs d'une organisation poursuivent des objectifs divergents et l'action collective n'est pas spontanée mais est un construit social s'articulant autour des intérêts des acteurs. L'individu au sein de l'organisation est un acteur autonome, à la liberté assurée mais aussi contrainte par l'incertitude. L'action collective nécessitant la coopération, la liberté d'un acteur reposera sur les zones d'incertitudes qu'il saura créer quant à la réalisation de sa tâche. Plus précisément, pour Crozier et Friedberg le pouvoir de A sur B dépend de la prévisibilité du comportement de $\mathrm{B}$ sur $\mathrm{A}$ et de l'incertitude où $\mathrm{B}$ se trouve du comportement de A. Tant que les besoins même de l'action créent des situations d'incertitudes, les individus qui doivent y faire face se trouvent disposer de pouvoir sur ceux qui seront affectés par les résultats de leurs choix. Les zones d'incertitudes créent un pouvoir et «ceux qui par 
leur situation, leurs ressources ou leurs capacités sont capables de contrôler (ces zones d'incertitudes), utiliseront leur pouvoir pour s'imposer face aux autres » (p.20). Dans ce contexte tout changement comme par exemple l'adoption d'une nouvelle technologie de l'information dans une organisation est l'occasion de faire évoluer les zones d'incertitudes et les relations de pouvoir entre individus. Concernant l'adoption d'une technologie de réseaux sociaux, les enjeux de pouvoir ne sont cependant pas uniquement liés à cette nouvelle manière de «jouer le jeu social de la coopération et du conflit » (Crozier et Friedberg, p. 30) qu'induit le changement. En donnant à voir les relations sociales et les positions de chacun dans l'organisation, les technologies de réseaux sociaux peuvent contribuer à réduire les zones d'incertitudes des acteurs. Pour les acteurs et dans l'objectif de conserver leur pouvoir, il y a donc un enjeu fort de maîtrise de la visualisation des relations sociales dans l'organisation. Cet enjeu est d'autant plus important que comme le souligne Bourdieu, le capital social peut être vu comme une des ressources permettant aux individus de conforter leur position sociale voire d'assurer leur domination au sein d'un champ (1994, 2000). Il définit différentes sortes de capital : le capital économique, le capital culturel, le capital social et le capital symbolique. Le capital économique fait référence à la richesse matérielle. Le capital culturel peut quant à lui se traduire à travers différentes formes tels que les biens culturels (tableaux, livres...), les connaissances et les compétences culturelles qui ont été inculquées à l'individu ou encore sous la forme de titres scolaires. Le capital social est lié au réseau de relations de l'individu. Le capital symbolique est ce que devient tout capital lorsqu'il est reconnu comme efficient par les autres acteurs (Perruchet et al., 2009). A partir du moment où le capital est perçu par les acteurs comme une force, un pouvoir ou un moyen d'exploitation, il est alors considéré comme capital symbolique et sa maîtrise permet d'assurer une domination au sein d'un champ social. Selon Bourdieu le capital symbolique est « une propriété quelconque, force physique, richesse, valeur guerrière, qui, perçue par les agents sociaux dotés des catégories de perception et d'appréciation permettant de la percevoir, de la connaître et de la reconnaître, devient efficiente symboliquement telle une force magique » (Bourdieu, 1994, p. 187) Posséder un capital symbolique permet de créer une légitimité acceptée par les autres acteurs sans remettre en question le caractère arbitraire de ce pouvoir. Dans un environnement organisationnel où le capital social est de plus en plus considéré comme un pré-requis à l'action collective, ce capital prend une dimension de capital symbolique pour les acteurs de l'organisation et le contrôle du développement de ce capital social est un enjeu déterminant pour établir la domination en organisation. L'introduction d'une technologie d'analyse des réseaux sociaux peut alors être une opportunité pour les acteurs de l'organisation de redistribuer le pouvoir et l'influence entre eux (Buckhardt et Brass, 1990). En rendant visible et en suscitant le développement du capital social au sein de l'organisation, les SNS peuvent permettre aux individus de faire évoluer les zones d'incertitudes et donc de faire évoluer leurs relations de pouvoir. Par ailleurs, parce que ces technologies sont susceptibles d'orienter le développement du capital social de l'organisation, leur contrôle peut permettre aux acteurs de conforter ou d'accroître leurs positions sociales.

En s'appuyant sur ce cadre théorique nous analysons dans cet article les enjeux organisationnels liés à l'appropriation d'un outil de réseaux sociaux au sein d'une organisation. Plus précisément nous cherchons à comprendre comment les enjeux de pouvoir liés au développement du capital social contribuent à l'appropriation de cette technologie. Pour mener cette recherche nous nous appuyons sur une démarche de Recherche Action au sein d'une collectivité territoriale. 


\section{Démarche de recherche : une Recherche Action au sein d'une collecti- vité territoriale}

Lorsqu'on se retrouve face à des situations trop complexes ou en constante évolution pour définir une problématique précise, ou face à un contexte renfermant des phénomènes sociaux ambiguës nécessitant la présence concrète du chercheur sur le terrain, la Recherche Action est alors la méthodologie de recherche la plus adaptée (Lewin, 1946 ; O'Brien, 1998 ; Baskerville, 1999; De Luca et al., 2008). Cette démarche consiste à introduire des changements au sein de l'organisation et à observer par la suite l'impact de ces changements en vue de les analyser pour enrichir le corpus théorique (Baskerville, 1999). La Recherche Action suppose alors plusieurs allers retours entre la pratique et la théorie. Cette interaction entre la théorie et le terrain permet d'approfondir pas-à-pas la compréhension d'un phénomène complexe. La démarche de Recherche Action est organisée selon trois phases : la phase de diagnostic (analyse du contexte de l'organisation étudiée en collaboration avec les acteurs de l'entreprise), la phase thérapeutique (introduction des éléments de transformation de l'organisation pour étudier leurs impacts) et la phase de développement des apprentissages (analyse du cas étudié et élaboration de «théories fondées ») (Baskerville, 1999).

L'objectif de notre recherche étant d'explorer une problématique émergente, la Recherche Action nous est apparue la méthode la plus adaptée (Lewin, 1946 ; Baskerville, 1999; Baskerville \& Pries-Heje, 1999). Nous menons cette recherche dans le cadre d'un projet de recherche et d'innovation financé par l'Etat français qui s'inscrit dans le plan de relance pour le développement de l'économie numérique. Ce plan de relance est un appel aux innovations technologiques 2.0 adaptées aux administrations publiques lancé au printemps 2008 . Le projet auquel nous participons vise à proposer aux collectivités territoriales un nouvel outil ainsi que de nouvelles pratiques de travail collaboratif fondées sur les réseaux sociaux des administrations. Il est mené en collaboration avec un fournisseur de solution informatique mettant à disposition un prototype de SNS. Pour mener cette Recherche Action nous sommes deux intervenants au sein de la Mairie avec pour rôle d'accompagner l'introduction de la technologie et de l'adapter aux besoins et usages du Service Public.

La Recherche Action s'est déroulée de Janvier 2010 à Mai 2011. Dès le démarrage de notre intervention à la Mairie, nous nous sommes présentés en tant que chercheurs adoptant une posture d'observateur-participant ayant deux objectifs :

- Accompagner l'adaptation de l'outil aux besoins des agents de la Mairie afin de s'assurer de son intégration dans les pratiques de travail ;

- Comprendre comment améliorer les processus de travail collaboratifs au sein des organisations publiques via l'intégration d'outils de réseaux sociaux.

Notre collaboration avec la Mairie passe par plusieurs formes d'interactions. Tout d'abord nous participons à des réunions bimensuelles en audioconférence depuis Janvier 2010 avec l'ensemble des partenaires pour échanger sur les avancées du projet et confronter les résultats. Nous menons également des échanges fréquents avec la Direction Logistique et Systèmes d'Information de la Mairie organisés en une réunion tous les deux mois. Enfin depuis Septembre 2010, nous participons à une réunion mensuelle avec un comité d'utilisateurs d'une douzaine de personnes. 


\section{Le cas du projet «Mairie et outil de réseaux sociaux»}

\subsection{Contexte du travail de recherche}

Le projet étudié consiste à intégrer une nouvelle technologie SNS (Social Network Systems) au sein d'une Mairie. Le SNS est un prototype développé par un centre de Recherche et Développement d'un fournisseur de solutions informatiques (voir Annexe). Il s'agit d'un outil qui permet d'analyser les profils ainsi que les relations existantes au sein d'une organisation dans le but de faciliter la recherche des compétences internes et la création des meilleures équipes possibles en fonction des projets à réaliser. Du fait de ses fonctionnalités, l'intégration de cette nouvelle technologie SNS a suscité l'intérêt de la Mairie et plus particulièrement de sa Direction Logistique et Systèmes d'Information. Son objectif est de développer des pratiques innovantes dans la gestion de ses procédures administratives internes et d'inciter à la collaboration interne au sein de la Mairie et notamment à la collaboration inter-services. Ce projet représente par ailleurs une opportunité pour la Mairie de se positionner comme ville pilote et créatrice de services innovants pour les citoyens et les autorités publiques. Enfin, la Mairie se veut aussi un support aux innovations technologiques des entreprises de sa commune et son implication dans ce projet l'aide à exprimer ce soutien.

La démarche de Recherche Action en relation avec la Mairie s'est déroulée en deux temps : une première phase de diagnostic avec l'objectif de comprendre le contexte de l'introduction de la technologie SNS au sein de la Mairie ; une deuxième phase thérapeutique d'accompagnement du déploiement de l'outil sur l'ensemble des agents.

\subsection{Phase de diagnostic : logiques d'action et culture technologique au sein de l'organisation}

Au démarrage de notre travail avec la Mairie, aucune annonce officielle concernant le projet n'avait été faite auprès des agents. Après plusieurs entretiens avec le Directeur Logistique et Systèmes d'Information en charge du déploiement du SNS et des échanges informels avec quelques représentants des Directions Fonctionnelles (Direction des Bâtiments notamment) nous avons tout d'abord identifié le contexte de l'introduction de l'outil.

\subsubsection{Spécificités de l'action collective au sein d'une Mairie}

L'organisation du travail au sein d'une commune française est régie par un cadre législatif strict reposant sur un pouvoir dual politique et administratif. Le Maire est élu au suffrage universel direct. Il nomme un conseil municipal qui a en charge de gérer des activités précises (Petite Enfance, Culture, Commerce...). Cette structure politique a pour rôle de donner les grandes orientations de l'action municipale. La mise en œuvre de cette action est orchestrée par une structure administrative parallèle. Le Maire recrute un Directeur Général des Services (DGS), fonctionnaire ayant le grade d'Administrateur Territorial. C'est le DGS en collaboration avec le Maire qui va animer la gestion de la politique de la ville. Pour cela il s'appuie sur des fonctionnaires recrutés avec l'aval du pouvoir politique. Ainsi chaque élu est aidé par un agent pour chaque grande politique de la ville. Toutefois aucun acte (dépense, recrutement...) ne peut être engagé par le personnel administratif. Seuls les élus ont ce droit.

D'un point de vue individuel l'agent de la fonction publique a des obligations particulières. Outre le respect du secret professionnel, l'agent de la fonction publique est tenu à une obligation de réserve mais aussi à une obligation de discrétion professionnelle d'information au public. De plus, l'action des organisations publiques est fortement assujettie à un ensemble de procédures formalisées ayant pour objectif la transparence vis-à-vis du citoyen et de l'Etat. De ce fait, les agents du secteur public sont contraints de conserver une traçabilité de 
toute action menée et de toute décision prise. Cet encadrement strict des pratiques des agents municipaux impacte négativement l'initiative et la mise en place de projets collaboratifs. Les agents ne disposent donc pas intuitivement d'une culture de collaboration.

Paradoxalement, les fonctionnaires travaillant dans une commune disposent pourtant d'une grande marge de liberté dans leurs actions. Dans la mesure où leur statut leur garantie la pérennité de leur emploi mais où s'ils sont aussi souvent résidents de la commune, ils sont également électeurs de la structure qui les gèrent. Aussi certains comportements irrationnels, émotionnels ou simplement de défense d'intérêts particuliers s'expriment-ils plus librement que dans le cadre d'une activité professionnelle gérée par le droit privé. Par exemple lorsqu'un agent ou une catégorie de personnel est soumis à un ordre venant de la hiérarchie et que cet ordre ne lui convient pas, il peut se sentir la liberté de passer outre la hiérarchie de la structure administrative pour s'adresser directement au Maire ou aux élus. Dans ce contexte l'action administrative nécessite d'associer fortement les agents aux décisions prises. L'objectif peut être parfois moins la recherche de la productivité que la recherche d'un consensus permettant l'action collective.

\subsubsection{Culture technologique de la Mairie}

$\mathrm{Au}$ sein de la Mairie avec laquelle nous travaillons, l'administration est organisée en plusieurs directions dont la Direction Logistique et Systèmes d'Information. Cette Direction est animée par un Directeur passionné, informaticien et disposant de la volonté de faire évoluer les pratiques de travail des agents de la Mairie. Avant le lancement du projet que nous étudions, la Direction Logistique et Systèmes d'Information a en particulier travaillé à la mise en place d'un projet d'intranet pour la Mairie. L'intranet est désormais opérationnel mais le projet a connu plusieurs blocages qui ont ralenti sa mise en service. Certains agents perçoivent en effet les outils informatiques comme difficiles à utiliser et requérant forcément des compétences techniques. Ils redoutent la difficulté qu'ils rencontreraient dans leur utilisation. D'autres agents, quant à eux, ont peur de la perte de contrôle sur les informations diffusées ou encore du risque que peuvent représenter les données partagées via les nouvelles technologies quant au respect de leur vie privée.

\subsubsection{Première adoption de l'outil}

Au démarrage du projet en Février 2010, le prototype SNS a été hébergé au niveau de la Direction Logistique et Systèmes d'Information. L'objectif étant d'adapter la technologie aux besoins de la Mairie, les informaticiens de la Direction Logistique et Systèmes d'Information se sont chargés des ajustements techniques et de son alimentation en données. Disposant d'une certaine autonomie et de compétences techniques en la matière, le Directeur Logistique et Systèmes d'Information a entièrement pris la main sur le nouvel outil pour mieux comprendre ses fonctionnalités et de quelle manière il peut contribuer à la collaboration interne et inter-services. Très rapidement, il se rend compte de toute la potentialité technique de cet outil collaboratif et de toute sa souplesse quant au traitement des données. Il décide alors de tester la flexibilité offerte par l'outil de réseau social en l'alimentant avec des données relatives à la gestion logistique de la Mairie (matériel bureautique, aux véhicules, budgets...) en allant jusqu'à faire des développements informatiques par lui-même et faire évoluer la version initiale fournie par le prestataire de la solution. Peu à peu l'outil, qui au départ devait être centré sur l'individu pour en faire un outil de collaboration, devient un vrai outil de contrôle de gestion et de monitoring qui permet de piloter efficacement les individus, les budgets, les coûts et les consommations de ressources. Cette vision de l'usage de l'outil est rapidement partagé par d'autres Directions Fonctionnelles comme la Direction des Bâtiments par exemple. 
A l'issue de cette première phase de diagnostic nous identifions donc deux logiques d'action qui interagissent et structurent les stratégies d'acteurs au sein de la Mairie. La première est une logique que l'on peut qualifier de «politique ». Elle est soutenue par la majorité des agents et vise à faire émerger l'action collective au travers d'un consensus permettant la satisfaction des intérêts particuliers. La seconde est une logique de gestion. Elle est portée en particulier par les Directions fonctionnelles (dont la Direction Logistique et Systèmes d'Information) et vise à développer l'action collective avec un objectif de productivité et d'efficacité.

Tableau 1. Les deux logiques d'action en interaction identifiées au sein de la Mairie à l'issue de la phase de diagnostic

\begin{tabular}{|l|l|l|}
\hline Porteurs & \multicolumn{1}{|c|}{$\begin{array}{c}\text { Logique d'action } \\
\text { «politique » }\end{array}$} & \multicolumn{1}{c|}{ Logique de gestion } \\
& $\begin{array}{l}\text { Les agents ayant une stabilité } \\
\text { de l'emploi et qui sont aussi } \\
\text { des électeurs pouvan in- } \\
\text { fluencer le pouvoir adminis- } \\
\text { tratif en passant par le poli- } \\
\text { tique. }\end{array}$ & $\begin{array}{l}\text { Les cadres et directions fonc- } \\
\text { tionnelles qui sont en charge } \\
\text { de la réalisation des procé- } \\
\text { dures internes et de la bonne } \\
\text { marche de l'action collective. }\end{array}$ \\
\hline Objectif visé & $\begin{array}{l}\text { Satisfaction des intérêts parti- } \\
\text { culiers des agents ou des } \\
\text { communautés d'intérêts. }\end{array}$ & $\begin{array}{l}\text { Satisfaction des usagers du } \\
\text { service public et réalisation } \\
\text { des objectifs collectifs fixés à } \\
\text { la collectivité territoriale. }\end{array}$ \\
\hline Forme & $\begin{array}{l}\text { La recherche du consensus } \\
\text { satisfaisant les intérêts parti- } \\
\text { culiers est préalable à toute } \\
\text { action collective et prime sur } \\
\text { la recherche de la productivi- } \\
\text { té. }\end{array}$ & $\begin{array}{l}\text { La recherche de l'amélioration } \\
\text { de la productivité et de } \\
\text { l'efficacité organisationnelle } \\
\text { est la priorité. }\end{array}$ \\
\hline $\begin{array}{l}\text { Rapport aux technolo- } \\
\text { gies }\end{array}$ & $\begin{array}{l}\text { Réticences liées à une non } \\
\text { familiarité avec ces outils, à } \\
\text { la crainte de la perte de con- } \\
\text { trôle des informations diffu- } \\
\text { sées. }\end{array}$ & $\begin{array}{l}\text { Intérêts liés à la possibilité } \\
\text { d'organiser } \\
\text { d'information et de les contrô- } \\
\text { ler. }\end{array}$ \\
\hline
\end{tabular}

\subsection{Phase thérapeutique : construire la convergence des logiques d'action}

Le démarrage de la phase thérapeutique correspond à la phase de déploiement de l'outil sur l'ensemble des agents de la Mairie. Les étapes clés de notre intervention sont résumées dans la figure 1.

\subsubsection{Le lancement du comité de pilotage}

A l'issue de la phase de diagnostic, nous avons suggéré à la Direction Logistique et Système d'Information d'introduire l'outil SNS non pas comme un outil de contrôle de gestion mais comme outil de collaboration pour revenir à l'objectif initial. Nous avons donc proposé de le présenter comme un annuaire étendu complémentaire à l'annuaire de la Mairie déjà existant en offrant notamment la possibilité d'avoir une vision globale sur qui fait quoi, qui 
travaille sur quels projets et sur l'organisation hiérarchique de la Mairie. Cette voie d'entrée nous est apparue efficace en termes d'intégration du nouvel outil car l'existence préalable de l'annuaire de la Mairie rend les nouvelles fonctionnalités plus familières et utiles et de ce fait devraient faciliter son appropriation par les agents. Elle semble offrir l'avantage de familiariser les acteurs avec la technologie et notamment les fonctionnalités de visualisation et d'analyse (qui fait quoi où ?) pour progressivement ouvrir aux fonctionnalités collaboratives (déclaration de réseau autonome et individuel).

Pour valider cette démarche et faire en sorte qu'elle réponde au mieux aux besoins des agents de la Mairie, des réunions mensuelles avec un comité de pilotage, composé d'une douzaine d'utilisateurs testeurs, ont été planifiées. Ces bêta testeurs ont été sélectionnés en concertation avec la Direction Logistique et Systèmes d'Information de la Mairie avec pour objectif d'associer très largement les agents au paramétrage de l'outil. Des acteurs clefs ont été mobilisés, représentant les partenaires sociaux (Syndicat UNSA, Syndicat CGT, Syndicat CFTC) ou des Directions Fonctionnelles de la Mairie telles que la Direction Générale des Services, la Direction des Ressources Humaines, la Direction de la Communication Interne et la Direction des Bâtiments.

\subsubsection{Le consensus des projets transversaux}

La première réunion avec le comité de pilotage a eu lieu en Novembre 2010. Chacune des entités mobilisées ont été représentées. Nous avons assuré l'animation de cette réunion.

Les potentialités de l'outil ont été rapidement comprises par les membres du comité de pilotage et les craintes vis-à-vis des technologies de l'information et de leur technicité n'ont pas été évoquées. Chaque membre du groupe de travail s'est rapidement construit une compréhension des fonctionnalités de l'outil. Cette compréhension a notamment conduit les membres du groupe de travail à évoquer les problèmes de «changement culturel» liés à la mise en place de cette technologie. Les membres du groupe de travail ont indiqué que l'outil appelait à "un changement de culture difficile à bouger», le blocage culturel étant attribué à la hiérarchie. Ainsi un représentant des partenaires sociaux a expliqué "Il faut que toute la hiérarchie s'ouvre à la transversalité. Il y a des verrous à faire sauter ».

Ensuite nous avons constaté que le débat a confronté les deux logiques identifiées dans la phase de diagnostic. Les agents présents et plus particulièrement les représentants syndicaux ont clairement exprimé leur objectif de défendre les intérêts individuels des agents. Une discussion longue sur la notion de compétence a ainsi mis en évidence la crainte de voir clarifier et afficher aux yeux de tous les diplômes et expériences des différents agents. La position des représentants syndicaux était qu'aucun élément de distinction entre agents quant à son engagement ou sa qualification professionnelle ne devait être mis en avant. Une représentante des partenaires sociaux a par exemple expliqué : "Pour avoir une vue d'ensemble, l'outil a une vraie plus-value. Mais les réserves que j'ai c'est par rapport à l'affichage de données personnelles. Je n'ai pas envie de voir mes compétences affichées. Comment caractériser la compétence? Qui pourrait décider de mon niveau de compétences? » La logique gestionnaire s'est quant à elle aussi exprimée en mettant en avant que l'utilité de l'outil est corrélée à la pertinence et l'originalité des informations qu'il met à disposition. Les représentants des Directions fonctionnelles (Directions des Ressources Humaines, Direction des Bâtiments, Direction Logistique et des Systèmes d'information...) étaient essentiellement intéressés par des informations concernant tout type de ressources humaines et matérielles. Comme l'explique un de ces directeurs fonctionnels : "L'avantage avec cet outil, est qu'il est très souple et il a beaucoup de potentialités. On peut mettre un tas d'informations dedans. On peut mettre tout ce qu'on veut : des codes RH, codes DGA 
(Direction Générale Adjointe), le matricule de l'agent mais aussi des informations sur le budget, les véhicules... on peut vraiment faire beaucoup de choses avec SNS. »

Progressivement un consensus a émergé. Les échanges ont unanimement fait ressortir le besoin de l'outil SNS pour mieux gérer les projets transversaux. Les projets transversaux sont des projets montés en collaboration entre plusieurs directions de la Mairie. Ils nécessitent donc la désignation d'un chef de projet, d'une équipe spécifique et une allocation budgétaire propre. Ces projets transversaux n'ont pour le moment pas bien fonctionné au sein de la Mairie.

Suite à cette première réunion et pour s'assurer de la pertinence de chaque information présente dans l'outil, nous avons mené une démarche d'analyse des usages de l'outil SNS fondée sur l'analyse systémique et l'analyse fonctionnelle. L'objectif était de caractériser les usages de l'outil pour préciser les informations nécessaires à chaque usage. Cette démarche devait permettre à chaque membre du groupe de travail de mieux comprendre pourquoi il est important de mettre à disposition telle ou telle information dans le SNS. Les résultats de cette analyse ont été adressés aux membres du groupe de travail sous la forme d'un document avant la réunion du mois de décembre. Lors de cette réunion, les débats ont été longs pour chaque information proposée. Par exemple une discussion a eu lieu sur la nécessité de mentionner le grade et la catégorie de chaque agent. Cette information pouvait être utile pour composer des groupes de travail transversaux. Toutefois il a été décidé que cette information ne devait pas être visible pour chaque agent mais seulement pour la hiérarchie. A l'issue de cette réunion, les usages ainsi que les informations à introduire dans l'outil pour le suivi des projets transversaux ont été validés. La Direction Logistique et Systèmes d'Information a été chargée d'intégrer l'ensemble des informations mentionnées dans l'outil afin de lancer un bêta test.

4.3.3. Collaboration avec le projet transversal «Dématérialisation» : La question de la responsabilité des actes de gestion

Afin d'accompagner l'intégration de l'outil dans les pratiques de travail des projets transversaux, en concertation avec le Directeur Logistique et Systèmes d'Information, nous avons été mis en relation avec un membre du comité de pilotage, responsable d'un projet transversal dit «Dématérialisation ». Ce projet a pour objectif de travailler à la numérisation de certaines procédures de travail au sein de la Mairie et à l'amélioration de la collaboration numérique inter-services. Aussi définir la démarche d'accompagnement d'un outil devant permettre de faciliter les pratiques de collaboration était susceptible de rentrer dans le périmètre des compétences de ce projet.

Pendant près d'un mois et demi des pourparlers ont eu lieu avec le chef de projet afin de voir comment travailler ensemble sur ce sujet. L'idée était de faire un état des lieux des pratiques collaboratives dans la Mairie pour voir comment intégrer le SNS pour soutenir ces pratiques collaboratives notamment pour les projets transversaux. Toutefois, même si le chef de projet a donné l'impression d'être intéressé à titre personnel par la démarche, un blocage est très vite apparu quant à la responsabilité de la réalisation de cette étude. Le chef de projet nous a indiqué que pour lui la responsabilité de cette étude ne pouvait pas lui incomber mais qu'elle relevait de la Direction Logistique et Systèmes d'Information. Comme il le dit "Comme ça, de manière très spontanée, il me semble en effet pertinent qu'une réflexion soit menée sur les pratiques de travail collaboratives, et (surtout) pas seulement technologiques d'ailleurs. En revanche, sur cette dernière question, il me semble qu'autant nous pouvons, au titre du groupe dématérialisation, vous apporter nos témoignages et ressentis etc. en la matière, en groupe « ressources » en d'autres termes, autant cet "audit » 
me semble devoir porter sur la manière dont la Direction Logistique et Système d'Information envisage les choses à cet égard. Formulé autrement, il me semble que la logique de votre démarche devrait être inversée, votre interlocuteur étant la Direction Logistique et Système d'Information et un compte rendu devant nous être adressé afin que nous puissions caler notre démarche avec le SNS mais également avec tous les autres projets menés par la Direction Logistique et Système d'Information. »

A l'inverse, la Direction Logistique et Systèmes d'Information a considéré qu'il n'était pas de son ressort d'accompagner le déploiement de l'outil et d'évaluer l'impact de l'outil sur les pratiques de travail collaboratives. Durant cette période nous avons donc identifié que la question de la responsabilité des actes de gestion est cruciale et préalable à toute action au sein de l'organisation. La thématique de l'introduction de pratiques de travail collaboratives via un outil est une question transverse à l'organisation qui ne rentre dans le mandat d'aucune structure existante. Les acteurs refusent donc d'assumer une responsabilité qui n'est pas actée.

\subsubsection{Présentation du prototype}

Une nouvelle réunion du comité d'utilisateur a eu lieu au mois de Mai 2011 avec pour objectif de présenter les évolutions techniques de l'outil et le premier prototype intégrant les informations discutées.

Lors de cette réunion, la discussion s'est peu à peu éloignée de la question de l'usage du SNS pour les projets transversaux pour se centrer sur la prise en main de l'outil SNS comme annuaire étendu complémentaire à celui déjà existant à la Mairie. Les partenaires sociaux ont adopté une posture défensive et revendicatrice sur l'outil qui s'est opposée à la logique de gestion des directions fonctionnelles. Des discussions sur les informations à présenter dans le profil agent et sur le périmètre d'accès aux informations dites sensibles de ce profil ont eu lieu dans un climat tendu. Puis les partenaires sociaux ont proposé de faire d'abord tester l'outil par le comité de pilotage. Or comme ces partenaires sociaux ne disposent pas toujours d'ordinateurs dans le cadre de leur activité professionnelle et ce même s'ils revendiquent d'être équipés au titre de leur activité syndicale, ils ont vu dans ce test l'occasion de satisfaire cette revendication exprimée de longue date. Ceci a abouti à une confrontation avec le Directeur Logistique et Systèmes d'Information qui a menacé d'arrêter le projet : "vous ne pouvez pas avoir des moyens informatiques (pc, réseaux) car vous n'êtes pas rattaché pleinement à la Mairie dans vos activités d'organisations syndicales. Jusqu'à présent le Directeur Général des Services ne vous a pas donné d'ordinateur, ce n'est pas à moi de le faire. Si on doit arrêter le projet pour cela, on l'arrête. ».

Après un débat virulent, le Directeur des Ressources Humaines a permis de faire converger les deux logiques d'action. Il a proposé une solution intermédiaire consistant à mettre à disposition deux ou trois ordinateurs en interne à la ville où les représentants syndicaux pourront accéder au logiciel pour le tester. En faisant cela il confirme la posture de conciliateurpédagogue qu'il a adopté dès le début de la réunion en expliquant par exemple la place du SNS par rapport aux autres outils et démarches de gestion des ressources humaines (annuaire actuel, gestion prévisionnelle des emplois et des compétences...) ou en plaidant pour un accompagnement spécifique de l'intégration de l'outil dans les pratiques de travail qu'il propose de prendre lui-même en charge. Cette intervention du représentant de la Direction des Ressources Humaines a été bien acceptée car au sein de cette Mairie, la Direction des Ressources Humaines bénéficie d'une légitimité reconnue et bien acceptée par l'ensemble des agents et que les autres Directions notamment la Direction Logistique et Système d'Information peinent à avoir. Ceci explique aussi le fait que pendant cette réunion, le re- 
présentant de la Direction Logistique et Système d'Information laisse volontiers le représentant de la Direction des Ressources Humaines prendre les devants aux moments des confrontations pour bénéficier de sa légitimité à faire converger un consensus vers une solution qui est souvent bien accueillie par les agents. Aussi à l'issue de cette réunion, la responsabilité du déploiement de l'outil a progressivement été prise en charge par la Direction des Ressources Humaines, soutenue par la Direction de la Communication et appuyée par la Direction de la Logistique et des Systèmes d'Information. Il s'agit là du signe d'un ancrage progressif de l'outil dans les pratiques de travail. La Direction des Ressources Humaines est perçue comme un service puissant au sein de la Mairie. L'implication clairement affichée de cette Direction dans le projet indique que l'outil entre progressivement dans la problématique des pratiques de travail de la Mairie.

\section{Figure 1. Les étapes clés de la phase thérapeutique}

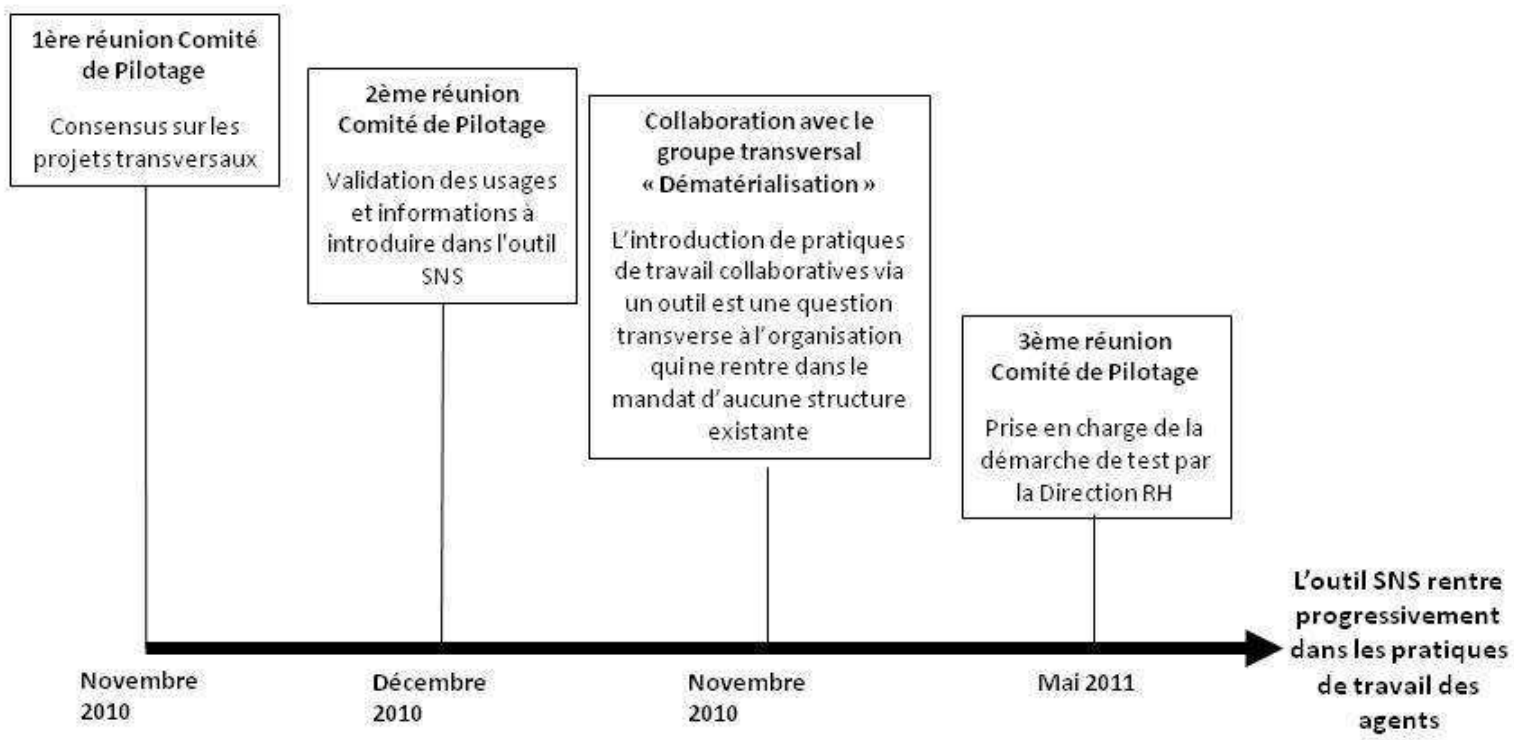


Tableau 2. Synthèse des observations recueillies à chaque phase de déploiement de l'outil SNS

\begin{tabular}{|c|c|}
\hline $\begin{array}{l}\text { Phases du déploiement de } \\
\text { l'outil }\end{array}$ & Observations \\
\hline $\begin{array}{l}\text { Lancement du comité de pilo- } \\
\text { tage avec proposition de l'outil } \\
\text { SNS comme complément à } \\
\text { l'annuaire Intranet }\end{array}$ & $\begin{array}{l}\text {-Technologie bien accueillie : pas de réticences à la } \\
\text { technologie exprimées en tant que telles } \\
\text { - Problème du nécessaire changement culturel à effec- } \\
\text { tuer évoqué par les membres du comité de pilotage } \\
\text { - Confrontation des deux logiques d'action identifiées } \\
\text { lors de la phase de diagnostic } \\
\text { Consensus sur l'introduction du SNS comme annuaire } \\
\text { étendu pour les projets transversaux }\end{array}$ \\
\hline $\begin{array}{l}\text { Le consensus des projets trans- } \\
\text { versaux : Paramétrage de } \\
\text { l'annuaire étendu pour les pro- } \\
\text { jets transversaux }\end{array}$ & $\begin{array}{l}\text {-Usages et logique des droits d'accès acceptés } \\
\text { - Confrontation des deux logiques d'action identifiées } \\
\text { lors du choix des informations à rentrer dans le SNS } \\
\text { pour le profil des agents }\end{array}$ \\
\hline $\begin{array}{l}\text { Collaboration avec le projet } \\
\text { transversal «Dématérialisa- } \\
\text { tion » : Etudier l'état des pra- } \\
\text { tiques collaboratives et la place } \\
\text { des outils de collaboration au } \\
\text { sein de la Mairie }\end{array}$ & $\begin{array}{l}\text {-Nécessité de clarifier la question de la responsabilité } \\
\text { des actes de gestion avant toute action au sein de la } \\
\text { Mairie } \\
\text {-Réticences autour d'un travail sur la thématique de la } \\
\text { collaboration, des pratiques de travail collaboratives et } \\
\text { des outils de travail collaboratifs dues au fait que cette } \\
\text { thématique est transverse à toute l'organisation et ne } \\
\text { rentre pas pour le moment dans le mandat d'une quel- } \\
\text { conque entité }\end{array}$ \\
\hline Présentation du prototype & $\begin{array}{l}\text {-Technologie bien accueillie et rapidement comprise } \\
\text { (nœuds, liens ascendants, liens descendants...) } \\
\text {-Affrontement des deux logiques d'action identifiées } \\
\text { sur : } \\
\text { a) le périmètre d'accès aux informations dites sen- } \\
\text { sibles du profil agent } \\
\text { b) les informations à présenter dans le profil agents } \\
\text { c) les modalités de test de l'outil } \\
\text { Consensus sur ces trois points construit par le repré- } \\
\text { sentant de la Direction des Ressources Humaines qui } \\
\text { choisit d'assumer la responsabilité du test de l'outil }\end{array}$ \\
\hline
\end{tabular}




\section{Analyse des apprentissages : caractéristiques de l'appropriation de l'outil à travers le prisme de la lutte pour l'appropriation du capital sym- bolique}

En s'appuyant sur le cadre d'analyse de l'acteur stratégique de Crozier et Friedberg et de la lutte pour le maintien des positions sociales de Bourdieu, l'analyse de l'appropriation de l'outil SNS par les acteurs met en évidence deux phases: une phase de prise de conscience de la dimension symbolique du capital social et une phase de lutte pour l'appropriation de ce capital symbolique au travers du paramétrage de l'outil.

\subsection{Quand le capital social devient capital symbolique}

Ce cas montre en premier lieu que l'introduction du SNS a fait prendre conscience aux acteurs de l'importance nouvelle de la maîtrise du développement du capital social au sein de leur organisation.

Tout d'abord, le SNS étant un outil 2.0, intuitif, visuel et centré sur l'utilisateur final, il conduit à une compréhension immédiate de ce que peut être le capital social de l'organisation. Ainsi lors de la première réunion avec le comité de pilotage, nous avons commencé à décrire l'outil SNS en tant qu'outil de réseaux sociaux et à présenter toutes les fonctionnalités qu'il pouvait offrir si on l'intégrait en tant qu'annuaire de la Mairie. Dès les premières dix minutes de la présentation de l'outil, les membres du comité de pilotage ont clairement saisi ce qu'étaient les réseaux sociaux et le capital social. Ils ont également rapidement compris les usages qui pouvaient être fait avec la technologie. Les agents ont compris par exemple qu'une fois alimenté en données concernant les profils et les compétences de chacun, l'information étant très visuelle serait alors facile d'accès et pourrait être utilisée notamment par les responsables hiérarchiques. Ils ont identifié l'enjeu que cet annuaire peut avoir en termes d'évaluation et de transparence concernant les acquis et expériences de chacun, la transparence conduisant naturellement à la diminution des zones d'incertitudes entourant l'action de l'agent et donc le pouvoir de chaque agent. Ils ont alors exprimé leurs réticences par rapport à cet outil susceptible de faire entrer chaque agent dans une logique d'évaluation individuelle affichée aux yeux de tous. Les directions fonctionnelles ont de leur côté compris que l'outil étant flexible et ergonomique, il pouvait être facilement utilisé pour mieux contrôler l'activité de l'organisation. Par exemple avec le premier paramétrage de l'outil comme outil de contrôle de gestion, on constate que la Direction Logistique et Systèmes d'Information est tentée d'utiliser l'outil pour exploiter le capital social des agents comme moyen de diminuer les zones d'incertitudes entourant leurs tâches (contrôle de l'individu et des ressources qu'il mobilise pour effectuer sa tâche).

En parallèle à cette prise de conscience de l'importance du capital social, on constate une prise de conscience progressive des potentialités de changements organisationnels suscitées par le déploiement de la technologie. Ainsi dès la présentation de l'outil, les agents évoquent que l'outil appelle à un changement culturel des pratiques de travail. Ils appellent de la sorte à redéfinir les zones d'incertitude et l'état du jeu social de la Mairie et cherchent à en tirer profit. L'exemple le plus significatif sur ce point est la tentative des partenaires sociaux d'exploiter le test de l'outil pour obtenir des ordinateurs utiles à leurs activités syndicales. Les directions fonctionnelles et notamment la Direction Logistique et Systèmes d'Information identifient également que l'introduction de la thématique du capital social via cette technologie va bousculer les périmètres de chaque entité et remettre en cause les zones d'incertitudes. Les réticences ou les craintes évoquées par les directions fonctionnelles (Direction des Systèmes d'Information et projet Dématérialisation) autour de la prise en charge de la responsabilité de l'introduction globale de l'outil (accompagnement du développement des pratiques collaboratives via l'outil SNS) évoquent une gêne vis-à-vis de 
l'émergence d'une problématique qui va fortement remettre en cause le périmètre de chacun et pourrait de ce fait conduire à une redéfinition du jeu social.

Ensuite plus symboliquement, lorsque les agents ont vu que non seulement il existait une technologie qui avait pour objectif de gérer des réseaux sociaux de l'organisation mais qu'un fournisseur de solution et des partenaires de recherche publics étaient impliqués dans son déploiement, ils ont compris l'importance qui était désormais attribuée au capital social par l'organisation. C'est probablement ce qui explique que tous les agents impliqués dans le comité de pilotage se sont libérés pour être présents lors de chaque réunion mensuelle.

Le déploiement du SNS fait donc prendre une dimension symbolique au capital social : le capital social devient un enjeu de pouvoir pour les acteurs de l'organisation et ouvre à une lutte pour son appropriation.

\subsection{La lutte pour l'appropriation du capital symbolique}

Au travers de ce cas, on constate que l'appropriation de la technologie SNS passe ensuite par une phase de lutte pour l'appropriation du capital social via le paramétrage de l'outil. La maîtrise du capital social via la technologie est perçue comme un élément qui permettra de dominer dans l'organisation.

Les potentialités des technologies 2.0 en termes de flexibilité et de personnalisation permettent aux acteurs de développer une bonne maitrise technique de l'outil pour orienter le développement du capital social dans un sens servant leurs intérêts et confortant leur position sociale dans l'organisation. Chaque acteur fait en sorte de détourner l'outil vers ce qui sert le plus ses intérêts. Ceci a été particulièrement perceptible lors de la discussion sur la nature des informations à intégrer dans l'outil. Lors de cette discussion chaque type d'information à mentionner dans l'outil (grade, service...) a été commenté, discuté et évalué quant à sa pertinence par chacun des membres du groupe de travail. Le choix de telle ou telle information à mentionner dans l'outil oriente le développement du capital social. Aussi assiste-t-on à un affrontement sur la maîtrise du développement du capital social autour des deux logiques identifiées (logique de gestion et logique politique), chacune cherchant à dominer l'autre.

Toutefois s'il y a recherche de la domination, la régulation de cet affrontement se fait bien. Si l'affrontement peut être virulent, chaque acteur est enclin à la recherche du consensus. Le premier est celui des projets transversaux. Orienter l'outil vers les projets transversaux représente une bonne opportunité pour parer aux menaces que chaque logique représente pour l'autre sans pour autant bloquer le projet d'intégration du SNS au sein de la Mairie qui apparaît comme innovant et donc porteur en matière de développement de zones d'influence pour chacune des logiques. Les projets transversaux n'ont pas pu se développer pleinement au sein de la Mairie. C'est donc un périmètre d'action ne présentant pas trop de danger pour évaluer comment tirer partie au mieux de l'exploitation du capital social via cet outil. Le choix d'orienter l'outil autour des projets transversaux permet aux acteurs de travailler autour d'un consensus offrant la possibilité à chaque partie prenante de se familiariser plus précisément avec les potentialités de l'outil en termes de développement du capital social. La lutte pour l'appropriation du capital symbolique qu'est le capital social via la technologie SNS a bien lieu mais passe par des phases de consensus.

Durant notre étude nous avons constaté que chaque logique souhaite prendre le dessus mais ne cherche pas à casser l'autre logique d'action ou la faire disparaitre. L'environnement de la Mairie est un environnement où les deux logiques politique et gestionnaire sont constamment en confrontation. L'équilibre social de l'organisation se construit autour de cette confrontation des deux logiques qui trouve sa résolution dans des consensus ponctuels permettant de mener une activité organisationnelle. Toutefois dans le cadre de notre étude 
l'arrivée d'un nouveau capital, susceptible de changer le jeu de domination, déstabilise l'organisation. Les acteurs ne sont pas familiers avec cette notion de capital social. Ils voient bien l'enjeu de la maîtrise de cette nouvelle ressource et identifient comment le capital social peut devenir capital symbolique et source de domination dans l'organisation. Pourtant n'étant pas parfaitement au fait des implications de l'utilisation de cette nouvelle ressource, ils adoptent un comportement attentiste vis-à-vis de l'utilisation de ce nouveau capital comme source de domination dans l'organisation. Le fait que lors de la réunion du mois de Mai 2011, en plein affrontement, l'intervention du représentant de la Direction des Ressources Humaines a conduit à immédiatement faire émerger le consensus peut mener à cette analyse. Au vue du niveau élevé de tension, ce revirement était particulièrement surprenant. Cette situation peut être interprétée comme l'acceptation d'une trêve dans l'affrontement pour s'emparer de la nouvelle ressource afin de prendre le temps de mieux comprendre ce qu'est vraiment ce nouveau capital symbolique.

Ainsi dans le cas étudié, l'appropriation de la technologie de réseaux sociaux passe par une phase d'appropriation socio-politique de la nouvelle ressource gérée (comment la nouvelle dimension donnée au capital social peut-elle servir ou gêner les intérêts de l'acteur ?), suivie d'une phase d'appropriation socio-politique de l'objet de gestion de cette ressource qu'est la technologie (comment la technologie peut-elle servir ou gêner les intérêts de l'acteur ?). Concrètement dans le cas étudié, cette appropriation se traduit par un processus structuré autour de phases d'affrontements suivies de phases de consensus. L'introduction de la technologie SNS contribue de la sorte à une appropriation du capital social en tant que nouveau capital symbolique pour l'organisation. 
Tableau 3. Analyse de l'appropriation de la technologie au travers du prisme de la théorie de l'acteur stratégique et de la théorie de la pratique

\begin{tabular}{|c|c|}
\hline Phases d'appropriation & Analyse de la situation \\
\hline $\begin{array}{l}\text { Phase 1. Le capital social devient } \\
\text { capital symbolique }\end{array}$ & $\begin{array}{l}\text { 1. Le SNS est un outil 2.0, tourné vers } \\
\text { l'utilisateur final, visuel, intuitif et flexible } \\
\text { >> Les acteurs prennent conscience de ce qu'est } \\
\text { le capital social } \\
\text { >> Les acteurs prennent conscience de ce que } \\
\text { l'organisation peut faire du capital social en utili- } \\
\text { sant la technologie } \\
\text { >> Les acteurs prennent conscience des potentia- } \\
\text { lités de changements organisationnels suscitées } \\
\text { par la mise en place de la technologie (possibilité } \\
\text { de faire bouger les zones d'incertitude) } \\
\text { 2. Une technologie existe pour gérer le capital so- } \\
\text { cial et l'accompagnement est prévu et pris en } \\
\text { charge } \\
\text { >> Les acteurs prennent conscience que le capital } \\
\text { social est important pour l'organisation }\end{array}$ \\
\hline $\begin{array}{l}\text { Phase } 2 \text {. Lutte pour l'appropriation } \\
\text { du capital symbolique }\end{array}$ & $\begin{array}{l}\text { Confrontation des deux logiques } \\
\text { d'action } \\
\text { Trêve dans l'affrontement/ } \\
\text { Consensus } \\
\text { n> Démarche spécifique d'appropriation du } \\
\text { l'appropriation de la technologie }\end{array}$ \\
\hline
\end{tabular}




\subsection{Synthèse de la démarche de recherche}

Etant donné notre objectif de recherche qui vise à identifier comment les acteurs s'emparent d'une technologie de réseau social dans un environnement organisationnel et la place que tiennent les enjeux socio-politiques dans l'appropriation d'un tel outil, nous avons organisé l'ensemble de nos interventions au sein de la Mairie autour des deux principales phases de la Recherche Action. La phase de diagnostic est une phase nécessaire à la compréhension du fonctionnement interne de l'organisation. Elle nous a permis de nous familiariser avec le contexte organisationnel, de rentrer en contact avec la Direction Logistique et Systèmes d'Information et de planifier nos interventions. A l'issue de cette phase, nous avons identifié deux logiques d'action en affrontement et nous avons pu caractériser la culture technologique des agents de niveaux hiérarchiques différents. Durant la phase thérapeutique et en collaboration avec la Direction Logistique et Système d'Information nous avons constitué un comité de pilotage réuni une fois par mois durant notre intervention. L'objectif de l'ensemble de ces réunions a été de mettre en œuvre un plan d'action qui tienne compte des besoins des agents représentés par le comité de pilotage et de réussir ainsi l'intégration de la technologie dans la pratique de travail des agents. La collaboration avec le comité de pilotage a abouti à la construction du premier prototype de l'outil SNS. Lors de la troisième et dernière phase consacrée à l'analyse des apprentissages, nous nous sommes penchés sur la nature de l'articulation entre les enjeux de pouvoir et l'appropriation de la nouvelle technologie par les agents. Pour ce faire nous nous sommes appuyés sur un cadre théorique fondé sur la théorie de l'acteur stratégique de Crozier et Friedberg (1977) et la théorie de la pratique de Bourdieu (1994 : 2000). La confrontation du cas au cadre théorique a montré que l'appropriation de l'outil SNS passe essentiellement par deux phases socio-politiques. Dans la première phase via l'outil l'acteur prend conscience de l'intérêt de la nouvelle ressource gérée (le capital social) et le capital social devient un capital symbolique au sens de Bourdieu. Dans une seconde phase l'acteur cherche à influencer la technologie (notamment via son paramétrage) pour qu'elle permette une exploitation de la ressource qui serve au mieux ses intérêts. Ci-dessous le tableau 4 récapitule les étapes de notre démarche de recherche. 
Tableau 4. Synthèse de la démarche de recherche

Phase 1- Diagnostic

\section{A. Objectif de la recherche}

a) Objectif théorique : comprendre dans quelle mesure les enjeux de pouvoir liés au développement du capital social contribue à l'appropriation d'une technologie de réseaux sociaux

b) Objectif appliqué : déployer une technologie de réseaux sociaux au sein d'une collectivité territoriale afin d'améliorer les processus de travail internes (notamment les processus de collaboration)

\section{B. Hypothèse de départ}

Les enjeux de pouvoir lié au développement du capital social impactent le processus d'appropriation d'une technologie de réseaux sociaux.

\section{Objectif de la phase de diagnostic}

Comprendre le contexte organisationnel de la Mairie et appréhender la culture technologique de l'organisation

\section{Recueil des données}

- Lectures sur le fonctionnement et le mode d'organisation des collectivités territoriales et Mairies françaises

- Echanges avec la Direction Logistique et Systèmes d'Information et d'autres Directions Fonctionnelles (Direction des Bâtiments notamment) dans le cadre de réunions de projet bi-mensuelles et dans le cadre de réunions seul-à-seul tous les deux mois.

\section{E. Résultats}

Identification de deux logiques d'action en interaction à la Mairie et de deux rapports différents aux technologies de l'information et de la communication

Phase 2- Thérapeutique

\section{A. Planification de l'action}

Objectif de la phase thérapeutique : Intégrer la technologie dans la pratique de travail des agents

En collaboration avec le Directeur Logistique et Système d'Information, mise en place :

- d'un comité de pilotage du déploiement du SNS (1 réunion par mois)

- d'une démarche d'intégration du SNS passant pour une première phase d'introduction du SNA comme annuaire complémentaire à l'annuaire actuellement existant

Livrables produits :

- Lettre de mission signée par le Directeur Général des Services et informant les membres du comité de pilotage de leur implication dans le projet et du périmètre du projet

- Présentation du prototype

\section{B. Mise en ouvre de l'action}

Travail avec les membres du comité de pilotage sur le développement du prototype (co-conception de l'usage)

Livrables produits :

-Analyse des usages de la technologie

-Paramétrage de l'outil 


\begin{tabular}{l} 
C. Evaluation de l'action \\
Via une analyse de contenu, le processus d'appropriation de l'outil est analysé pour évaluer le rôle \\
des enjeux de pouvoir liés au développement du capital social sur le processus d'appropriation de \\
la technologie. \\
Données analysées : \\
-Réunions du comité du pilotage \\
-Emails échangés avec les membres du comité de pilotage \\
-Réunions téléphoniques avec les membres du comité de pilotage \\
Résultats : \\
-L'hypothèse de travail est validée : les enjeux de pouvoir liés au développement du capital social \\
impactent bien le processus d'appropriation de la technologie \\
- La technologie s'intègre progressivement dans les pratiques de travail (prise en main de son dé- \\
ploiement par la Direction des Ressources Humaines) \\
\hline Phase 3. Analyse des apprentissages \\
\hline A. Objectif \\
Approfondir la compréhension du lien enjeux de pouvoir liés au développement du capital social et \\
appropriation de la technologie par les acteurs \\
B. Méthode \\
Confrontation des résultats de l'analyse avec la théorie de l'acteur stratégie (Crozier et Friedberg, \\
1977) et la théorie de la pratique (Bourdieu, 1994; 2000) \\
C. Résultat \\
L'appropriation de la technologie SNS passe par une phase d'appropriation socio-politique de la \\
nouvelle ressource gérée par la technologie (comment le capital social peut-il servir ou gêner les \\
intérêts de l'acteur ?), suivie d'une phase d'appropriation socio-politique de l'objet de gestion de \\
cette ressource qu'est la technologie (comment la technologie SNS choisie et paramétrée peut-elle \\
servir ou gêner les intérêts de l'acteur ?).
\end{tabular}

\section{Discussion et conclusion}

Dans le contexte émergent de l'introduction des technologies de réseaux sociaux au sein des organisations, cette Recherche Action est tout d'abord une illustration des enjeux liés à l'introduction de telles technologies au sein des organisations.

Elle montre ainsi que l'introduction d'une technologie SNS peut avoir un impact significatif sur le développement du capital social dans une organisation. Le SNS semble faire évoluer la perception qu'ont les acteurs de la valeur de leur capital social. Le capital social a toujours existé au sein des organisations. Il a toujours été plus ou moins mobilisé par les acteurs. Toutefois l'émergence de ces nouveaux outils et leur introduction dans les organisations font prendre une nouvelle dimension au capital social. Le cas étudié montre que l'introduction d'une technologie dédiée à la gestion d'une ressource jusque là non valorisée par l'organisation conduit les acteurs à prendre conscience de la nouvelle valeur attribuée par l'organisation à la ressource. Cette prise de conscience de la valeur du capital social via l'introduction d'une technologie SNS peut conduire les acteurs à chercher à accroître leur capital social par le développement de leur réseau social et ce afin d'accroître leur pouvoir. 
Une des perspectives de ce travail est en premier lieu d'approfondir l'analyse de l'impact d'une technologie SNS sur le développement du capital social. Dans cette recherche l'outil n'est pas encore utilisé au quotidien par les acteurs. La lutte pour l'appropriation du capital social s'exprime dans le paramétrage de l'outil. La question se pose de savoir comment les acteurs vont au quotidien utiliser cette technologie pour développer leur capital social. Sur les réseaux sociaux externes aux organisations, les stratégies d'acteurs peuvent être très variées (Cardon, 2008). L'utilisation d'un SNS en interne à une organisation va-t-elle conduire naturellement les acteurs à mettre en avant leur capital social pour se promouvoir ou au contraire vont-ils chercher à le cacher pour qu'il ne puisse pas être contrôlé par l'organisation ? L'implémentation d'un SNS va-t-il en outre stimuler le développement du capital social des individus ou ne va-t-il que refléter un existant?

Ce cas met également en évidence le rôle que peuvent jouer les Systèmes d'Information dans le maintien ou le développement des positions sociales des acteurs de l'organisation. La facile prise en main de ces outils par les acteurs leur permet d'orienter très fortement leurs usages dans l'objectif de la satisfaction de leurs propres intérêts. Au-delà, la maîtrise de la technologie donnant à voir ce qu'est le capital social de l'organisation semble contribuer à l'affirmation du groupe d'acteurs dominant dans l'organisation. La maîtrise de l'outil SNS peut être perçue comme le signal et le symbole de la maîtrise du capital social de l'organisation (Feldman \& March, 1981). Dans le cas étudié nous constatons que le déploiement du SNS conduit effectivement à une phase d'appropriation des opportunités offertes par ce nouveau capital qu'est le capital social en termes de développement des positions sociales dans l'organisation. La Direction Logistique et Systèmes d'Information y voit par exemple une formidable opportunité d'accroître sa maîtrise des ressources et donc sa position dominante dans l'organisation. Ce travail appelle donc à un approfondissement des recherches sur l'utilisation des SI pour le maintien et le développement de positions sociales en organisations dans la lignée des travaux de Dudezert et Leidner (2011).

Enfin, ce cas ouvre à une réflexion sur les modèles d'appropriation des technologies. De Vaujany (2006) décrit le processus d'appropriation de la technologie du point de vue des utilisateurs comme passant par une phase psycho-cognitive nécessitant un apprentissage particulier pour la prise en main de l'outil suivie d'une phase d'appropriation sociopolitique permettant aux acteurs de comprendre comment les technologies peuvent gêner ou servir leurs intérêts. Dans le cas étudié nous observons que la phase d'appropriation psycho-cognitive est très courte voire quasi-inexistante. Les technologies 2.0 sont centrées sur l'utilisateur final, visuelles, flexibles et personnalisables (Boughzala, 2011) et semblent très rapidement appropriées par les acteurs du point du vue psycho-cognitif. En revanche, nous identifions que l'appropriation de la technologie par l'utilisateur passe d'abord par une phase consistant à s'approprier la nouvelle ressource gérée par la technologie (le capital social). Pour l'acteur il s'agit de comprendre l'intérêt que représente ce nouveau capital. Ensuite il s'agit de comprendre comment l'outil SNS déployé peut servir ou non ses intérêts en terme de maîtrise de ce nouveau capital. Ainsi la phase d'appropriation socio-politique de la technologie passerait à la fois par une phase d'appropriation socio-politique d'une nouvelle ressource et par une phase d'appropriation d'un nouvel objet de gestion de cette ressource qu'est la technologie. Une des perspectives de ce travail est d'approfondir cette étude du processus d'appropriation des technologies 2.0.

Ce travail de recherche s'appuie sur une Recherche Action d'un an et demi. Pour ce type de protocole de recherche, le critère de validité scientifique est le critère d'enseignabilité. La connaissance produite est scientifique si elle est reproductible, intelligible, et reconstructible. Pour satisfaire ce critère dans cet article nous avons décrits les finalités de la recherche, le contexte de la recherche et la démarche de recherche menée en interaction avec 
le terrain. Une synthèse de cette démarche est proposée dans la section 5.3. Toutefois cette recherche reste exploratoire et devra ouvrir la voie à des projets de recherche permettant une généralisation des conclusions. Notamment cette recherche a été menée dans le contexte spécifique des collectivités territoriales françaises. Ces organisations sont aujourd'hui en pleines mutations et évoluent de structures bureaucratiques à des structures plus flexibles, plus proches des structures post-bureaucratiques (introduction du mode projet, travail en réseau avec les communautés d'agglomération, les conseils généraux, les conseils régionaux....). Cette étude permet ainsi d'apporter un éclairage sur le rôle des technologies dans la mutation des organisations d'un modèle hiérarchique à un modèle flexibilisé postbureaucratique. Cependant pour être généralisés, les résultats présentés ici devront être confrontés à d'autres types d'organisations (entreprises, associations...).

Pour conclure, ce projet de recherche nous a permis d'appréhender l'impact de l'introduction d'un outil de réseaux sociaux dans une organisation publique en identifiant les enjeux de pouvoir mis au cœur du processus de l'appropriation du SNS. Avec l'introduction de ce type de technologies dans une organisation, le capital social revêt une dimension symbolique qui lui donne de l'importance pour les acteurs de l'organisation. De ce fait il devient alors important pour les acteurs de maitriser ces outils afin de protéger leur capital social et de conforter ou d'accroitre leur pouvoir informel dans l'organisation. Ce travail contribue également à éclairer d'un nouveau jour la prudence des entreprises quant à l'introduction de ces technologies. Si les technologies de réseaux sociaux sont susceptibles d'accélérer et de flexibiliser les processus de travail internes à une organisation, les enjeux socio-politiques de leur appropriation nécessitent que leur déploiement s'appuie sur un accompagnement spécifique permettant de concilier les pratiques de travail collaboratives, le développement de relations informelles et le respect du cadre formel, planifié et hiérarchisé de l'organisation. 


\section{Annexe : Copies d'écran de l'outil SNS déployé}

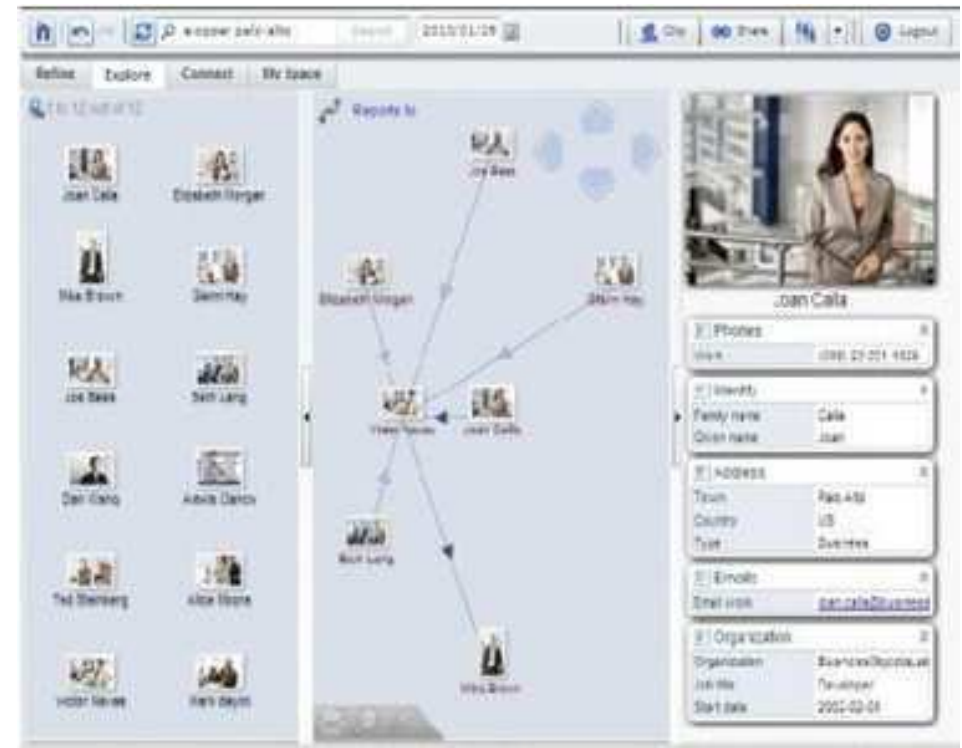

Figure 1: Capture d'écran du réseau d'un acteur au sein d'une organisation (tel qu'il apparait via l'outil SNS)

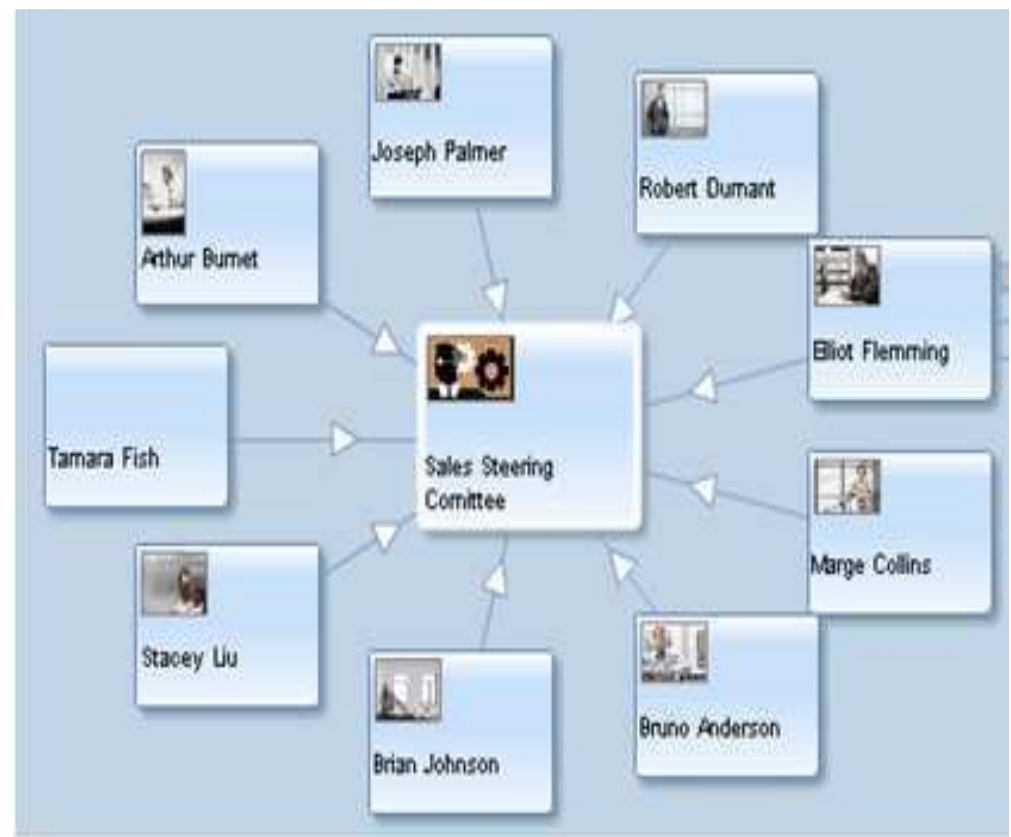

Figure 2: Capture d'écran de la composition d'une équipe projet (tel que ça apparait via le l'outil SNS) 


\section{Références}

Anklam P. (2003), "KM and the social network ", case study: KM and the Social Network.

Baskerville R.L. (1999), "Investigating Information Systems with Action Research", Communications of the Association for Information Systems, Vol. 2, $\mathrm{n}^{\circ} 19$.

Baskerville, R., et Pries-Heje, J. (1999), "Grounded Action Research: A Method For Understanding IT in Practice", Accounting, Management and Information Technology, ${ }^{\circ}$ 9, p. 1-23.

Borgatti S. P. et Foster P. C. (2003), "The Network Paradigm in Organizational Research: A Review and Typology ", Journal of Management, (29:6), p. 991-1013.

Boughzala I. (2011), "Collaboration 2.0 through the new organization transformation", in Knowledge Management 2.0: Organizational Models and Enterprise Strategies, ouvrage coordonné par I. Boughzala et A. Dudezert, IGI Publishing, Septembre 2011, p. 1-16.

Bourdieu P., (1980), "Le capital social. Notes provisoires ", Actes de la recherche en sciences sociales, vol. 31, janvier, pp. 2-3

Bourdieu, P., (1985), "The Forms of Capital ", in Handbook of Theory and Research for the Sociology of Education, J. G. Richardson (Ed.), Greenwood Press, New York.

Bourdieu, P., (1987), “Choses dites”, Minuit.,Paris.

Bourdieu P. (1994), "Stratégies de reproduction et modes de domination ", Actes de la recherche en sciences sociales, No. 105 , p. 3-12.

Bourdieu P. (1994), “Raisons pratiques : Sur la théorie de l'action", Editions du Seuil, Paris

Bourdieu P. (2000), "Les structures sociales de l'économie", Editions du Seuil, Paris

Boxman E.A.W., DeGraaf P.M., Flap H.D. (1991), "The impact of social and human capital on the income attainment of Dutch managers", Social Networks, ${ }^{\circ} 13$, p. 51-73.

Boyd, D.M., et Ellison, N.B. (2007), “Social Network Sites: Definition, History, and Scholarship”, Journal of ComputerMediated Communication, (13:1), p. 210-230.

Brass D.J. (1984), "Being in the right place: A structural analysis of individual influence in an organization", Administrative Science Quarterly, ${ }^{\circ} 29$, p. 518-539.

Buckhardt M. et Brass D. (1990), "Changing Patterns or Patterns of Change: The Effects of a Change in Technology on Social Network Structure and Power ", Administrative Science Quarterly, n 35, p. 104-127.

Burt, R. S. (1992), "Structural holes: The social structure of competition", Cambridge, MA: Harvard University Press.

Burt R. S. (2000), "Structural Holes versus Network Closure as Social Capital", Pre-print for a chapter in Social Capital: Theory and Research, edited by Nan Lin, Karen S. Cook and R. S. Burt. Aldine de Gruyter, 2001.

Burt, R. (2005)," Brokerage and Closure: An introduction to Social Capital", London: Oxford University press.

Burton P., Wu Y., Prybutok V. R. (2010), "Social Network Position and Its Relationship to Performance of IT Professionals", the International Journal of an Emerging Transdiscipline, $\mathrm{n}^{\circ}$. 13, p. 121-137.

Cardon D. (2008), "Le design de la visibilité: un essai de typologie du web2.0", InternetActu.net.

Coleman J. S. (1988), "Social Capital in Creation of Human Capital", The American Journal of Sociology, Vol. 94, Supplement S95-S120

Cross R., Parker A., Prusak L. (2000), "Knowing what we know: Supporting knowledge creating and sharing in social network", IBM Institute for Knowledge Management.

Cross R., Parker A., Borgatti S. P. (2002), "A bird's-eye view: Using social network analysis to improve knowledge creation and sharing", IBM Institute for Knowledge-Based Organizations.

Crozier M. et Friedberg E. (1977), "L'Acteur et le système", Le Seuil, Paris.

Deluca D., Gallivan M.J., et Kock N. (2008), "Furthering Information systems Action Research: A Post-Positivist Synthesis of Four Dialectics", Journal of the Association for Information Systems, (9: 2), p. 48-72

Deltour F., Plé L. et Roussel C.S (2011), " Knowledge sharing in the age of web 2.0 : A social capital perspective", in Knowledge Management 2.0: Organizational Models and Enterprise Strategies, ouvrage coordonné par I. Boughzala et A. Dudezert, IGI Publishing, Septembre 2011, p. 122-141. 
De Vaujany F. X. (2006), "Pour une théorie de l'appropriation des outils de gestion : vers un dépassement de l'opposition conception-usage", Management \& Avenir, (3 :9), p. 109-126.

Dudezert A., et Leidner D. (2001), "Illusions of control and Social Domination Strategies in Knowledge Mapping System Use", European Journal of Information Systems, n 20, p. 574-588.

Elie-Dit-Cosaque C. (2010), "Les réponses de cadres intermédiaires face à l'implémentation de technologies de l'information et de la communication : le cas d'une administration publique", Management et Avenir, (9: 39), p. 213-232.

Ehrlich K., Lin C.Y., Griffiths-Fisher V. (2007), "Searching for Experts in the Enterprise: Combining Text and Social Network Analysis ", Proceedings of the international ACM conference on Supporting group work.

Feldman M.S.et March J.G. (1981), "Information in Organizations as Signal and Symbol", Administrative Science Quarterly, (26:2), p. 171-186.

Fernandez R. M., Castilla E. J., Moore P. (2000), "Social Capital at Work: Networks and Employment at a Phone Center", American Journal of Sociology, (105: 5), p. 1288-1356.

Ferron, M., Massa, P., et Odella, F. (2011), “Analyzing collaborative networks emerging in Enterprise 2.0: the Taolin Platform”, Procedia - Social and Behavioral Sciences, $n^{\circ} 10$, p. 68-78.

Fisher D. et Dourish P. (2004), « Social and Temporal Structures in Everyday Collaboration », Conference on Human Factors in Computing Systems, April 24-29, Vienna, Austria.

Fuhrer C., Cucchi A., Picard P. (2009), "Les capacités relationnelles des technologies de l'information : le concept de capital technico-social a-t-il un sens ? ", Actes du $14{ }^{\text {ème }}$ Congrès de l'AIM, Marrakech, juin 2009

Lazega E. (2006), "Le capital social de l'organisation flexibilisée", Revue française de gestion, (4 :163), p. 127-137.

Lecko (2011), «Réseaux sociaux d'entreprise (Tome : 3) : Une disparité propice à une bulle de la valeur 2.0 », URL : http://www.lecko.fr/etude-reseaux-sociaux-d-entreprise-tome-3.html

Lewin K. (1946), "Action research and Minority Problems", Journal of Social Issues, (2:2), p. 34-46.

Manhes P. (2011), «Réseaux sociaux : la troisième ère de l'entreprise », Le Cercle Les Echos, Mars 2011, URL source: http://lecercle.lesechos.fr/cercle-entrepreneur/marketing-communication/221133768/reseaux-sociaux-troisieme-ereentreprise

Muhlmann D. (2001), "Des nouvelles technologies à l'image des vieilles organisations", Sociologie du travail, $\mathrm{n}^{\circ}$ 43, p. 327-347.

Nahapiet J. et Ghoshal S. (1998), "Social Capital, Intellectual Capital, and the Organizational Advantage ", The Academy of Management Review, (23: 2), p. 242-266.

Nov, O. (2009). "Chapter 1 Information Sharing and Social Computing: Why, What, and Where?, Advances in Computers, $\mathrm{n}^{\circ} 76$, p. 1-18.

O’Brien R. (1998), "An Overview of the Methodological Approach of Action Research", Retrieved from:http://www.web.net/ robrien/papers/arfinal.html

Pempek, T.A., Yermolayeva, Y.A. et Calvert, S.L. (2009), “College students' social networking experiences on Facebook", Journal of Applied Developmental Psychology, (30: 3), p. 227-238.

Pereira C. S. \& Soares A. L. (2007), "Improving the quality of collaboration requirements for information management through social networks analysis", International Journal of Information Management, n² 27, p. 86-103.

Perruchet A., Paul J.J., Lallement J. (2009), « Le doctorat: un investissement rentable? : approches économiques et sociologiques », Edition L'Harmattan.

Putnam R. D., (2000), “Bowling Alone: The Collapse and Revival of American Community”, Simon \& Schuster, New York.

Richter, D., Riemer, K. et Vom B. (2011), "Internet Social Networking", Business \& Information Systems Engineering, (3: 2), p. 89-101.

Roblyer, M.D., Webb M., Herman J. et Witty J.V. (2010), "Findings on Facebook in higher education: A comparison of college faculty and student uses and perceptions of social networking sites", The Internet and Higher Education, (13:3), p. 134-140.

Sparrowe R.T. et Liden R.C. (1997), "Process and structure in leader-member exchange", Academy of Management Review, (22: 2), p. 522-552.

Star, S.L., et Bowker, G.C. (1995), "Work and infrastructure", Communication of the ACM, (38:9), p. 41. 
Star, S.L. et Strauss, A. (1999), "Layers of Silence, Arenas of Voice: The Ecology of Visible and Invisible Work", Computer Supported Cooperative Work, $\mathrm{n}^{\circ}$ 8, p. 9-30.

Steinfield, C., DiMicco, J. M., Ellison, N. B., et Lampe, C. (2009), "Bowling Online: Social Networking and Social Capital within the Organization", Proceedings of the Fourth Communities and Technologies Conference.

Szulanski G. (1996), "Exploring internal stickiness: impediments to the transfer of best practice within the firm", Strategic Management Journal, $\mathrm{n}^{\circ} 17$, p. 27-43. 